\title{
Flexibilité de l'entreprise et implication des employés
}

Etude de cas d'un prestataire de sport et de tourisme

Business flexibility and employees involvement : a single case study of a company provider of sports and tourism-related services

\section{Élodie Paget et Jean-Pierre Mounet}

\section{OpenEdition}

\section{Journals}

Édition électronique

URL : http://journals.openedition.org/travailemploi/1539

DOI : 10.4000/travailemploi.1539

ISSN : 1775-416X

\section{Éditeur}

DARES - Ministère du Travail

\section{Édition imprimée}

Date de publication : 15 juin 2010

Pagination : 5-14

ISSN : 0224-4365

\section{Référence électronique}

Élodie Paget et Jean-Pierre Mounet, «Flexibilité de l'entreprise et implication des employés », Travail et Emploi [En ligne], 122 I avril-juin 2010, mis en ligne le 15 juin 2012, consulté le 19 avril 2019. URL http://journals.openedition.org/travailemploi/1539; DOI : 10.4000/travailemploi.1539 


\title{
Flexibilité de l'entreprise et implication des employés: étude de cas $d$ 'un prestataire de sport et de tourisme
}

\author{
Élodie Paget (*), Jean-Pierre Mounet (**)
}

Les variations cycliques et acycliques du secteur en mutation du sport et du tourisme exercent de fortes contraintes sur les organisations prestataires de services s'y insérant. À partir de l'analyse monographique d'une petite entreprise, l'article rend compte des stratégies de flexibilité développées afin de s'adapter à l'instabilité du marché. Mais il montre également comment ces pratiques de flexibilité spécifiques peuvent être acceptées par les "employés», qui constituent la principale variable d'ajustement, et même être vécues de manière positive. L'article évoque une distribution particulière des rôles au sein de l'entreprise laissant une large autonomie aux "employés» et favorisant leur implication dans le travail, ainsi qu'un brouillage des temps sociaux, notamment entre temps de travail et temps de loisir, participant à l'engagement des acteurs de l'entreprise dans le «projet» de croissance du dirigeant.

La réduction du temps de travail avec l'augmentation régulière des congés payés et la baisse de la durée légale du travail à 35 heures ont entraîné une réorganisation des temps sociaux. Ainsi, la société contemporaine a vu apparaître une montée du temps individuel (VIARD, 2006) faisant la part belle au loisir. Le développement de ce dernier, défini comme la part non contrainte de ce temps libéré (PRONOVOST, 1996), s'inscrit dans une légitimité acquise progressivement depuis les années 1970, en rupture avec l'éthique du travail et de la réussite qui prédominait jusque-là (Clément, Defrance, Pociello, 1994). Il correspond à l'émergence de nouvelles tendances socioculturelles induisant de nouvelles manières de voyager (CARNET DE ROUTE POUR LA MONTAGNE, 2001; URBAIN, 2002) et de nouvelles modalités de pratique physique et sportive (ChIFFLet, 1999; LEBLANC, 1992; Loret, 1995; Maurice, 1987; Pociello, 1995, 1999).

Le secteur du sport et du tourisme a connu ces dernières décennies de nombreuses transformations, et il est devenu un marché à part entière. Les entreprises du secteur, prestataires de services sportifs et touristiques, évoluent dans un environnement en mutation. Or, ces entreprises sont majoritairement des petites entreprises (Paget, Mounet, Gullhon,

(*) LAS-LARES - université Rennes 2, ZAC Atalante Champeaux, 3 allée Adolphe Bobierre, 35000 Rennes; elodie. paget@univ-rennes2.fr

(**) Laboratoire sport et environnement social - université Joseph Fourier Grenoble 1, UFR APS, BP 53, 38041 Grenoble Cedex 9; jean-pierre.mounet@ujf-grenoble.fr
2007), du point de vue de leur taille mais également de leurs spécificités de gestion: moins de cinquante salariés(1), un dirigeant centralisateur, une faible spécialisation des tâches, des systèmes d'information interne et externe simples, un environnement proche. Ainsi, pour survivre, se maintenir ou encore se développer lorsqu'elles ont un objectif de croissance, ces petites entreprises doivent tenir compte de cette instabilité du secteur. Un des moyens de régulation utilisés est la pratique de flexibilité. Cette dernière recouvre de multiples réalités (TARONDEAU, 1999), parmi lesquelles nous retiendrons, dans le cadre de cette étude, deux typologies souvent mobilisées par les chercheurs. La première distingue la flexibilité interne (apportée par les salariés, liée au temps de travail et aux modalités d'organisation du travail) de la flexibilité externe (consistant à mobiliser des acteurs ou des organisations situées dans l'environnement de l'entreprise, et portant sur les statuts d'emplois ou sur les systèmes de production). La seconde différencie la flexibilité quantitative (ajuster les volumes de travail et d'emploi: heures travaillées, nombre de salariés...) de la flexibilité qualitative (modifier les modes d'organisation du travail, les systèmes de production...). Mais si les pratiques de flexibilité ont déjà fait l'objet de nombreux travaux de recherche (BARBIER, NADEL, 2003; Beaujolin-Bellet, 2003, 2004 ; Bunel, 2006; Brunhes, KaIsergruber, 1997; Everaere, 1999; JACQUOT, SETTI, 2002; UlRICH, 2003), aucun à notre connaissance, ne s'est penché spécifiquement sur le

(1) Définition de la Commission européenne. 


\section{Encadré 1 \\ Méthodologie de la recherche}

Cette recherche a privilégié une démarche inductive (GLASER, StRAuss, 1968) et compréhensive (WEBER, 1992). Comme le souligne Bernoux (2004, p. 247), la connaissance d'une organisation «ne peut être fondée que sur l'observation attentive et la parole de ceux qui vivent les situations de travail ». Cette enquête part donc du discours des acteurs et du sens que ces derniers donnent à leurs actions pour reconstruire la réalité du système. Afin d'apporter un éclairage approfondi des logiques d'action à l'œuvre au sein du secteur sportif et touristique, cette recherche s'appuie sur une monographie.

\section{L'entreprise Delta, objet de la monographie}

Delta est une petite entreprise prestataire de sport et de tourisme, créée en 1998. Implantée dans les Alpes françaises, cette société connaît une forte croissance(1) et son métier revêt deux aspects. D'une part, l'entreprise est agent de voyages. Elle conçoit sur mesure des produits à forfait(2): l'entreprise assemble différentes prestations (hébergement, transport, restauration, activités...) qu'elle vend à un prix tout compris. Elle est spécialisée dans le tourisme d'affaires et organise à titre principal des séminaires(3). D'autre part, Delta est producteur d'activités. Elle réalise en interne des produits sportifs (4): l'entreprise possède en son sein les ressources nécessaires à la conception, l'organisation et la production de ces services. Delta propose ainsi toute une gamme de produits allant de la simple activité (sportive) à de l'assemblage de prestations pour former un produit touristique d'affaires.

Cette petite entreprise est une SARL composée de cinq associés (quatre principaux) dont le dirigeant associé majoritaire. Son capital social avoisine les 90000 euros et son chiffre d'affaires est d'environ 2,5 millions d'euros. Elle emploie cinq salariés en contrat à durée indéterminée et un en contrat à durée déterminée. La moyenne d'âge de ces employés est jeune, trois sont des femmes. Les seuls acteurs de l'entreprise de plus de 40 ans (masculins) sont le dirigeant, les associés et les travailleurs indépendants avec lesquels travaille régulièrement l'entreprise.

\section{Les méthodes d'investigation}

Selon YIN (2003), la validité des connaissances est conditionnée par le recours à au moins deux sources d'investigation différentes. Dans le cadre de cette recherche et dans une perspective qualitative, notre choix s'est porté sur l'utilisation de trois types de sources: observation participante, entretiens semi-directifs, documents. Cette approche multiméthodes (BREWER, HUNTER, 1989) a mené à «la construction d'une chaîne logique d'indices et de preuves" (Miles, Huberman, 2003, p. 31).

\section{L'observation participante}

L'observation participante doit être comprise comme une présence physique du chercheur (le premier auteur de cet article) au sein de l'entreprise Delta. Durant toute la période de ces travaux de recherche, il a fait ponctuellement partie de l'entreprise Delta, sur plusieurs périodes allant de quelques jours à quatre mois consécutifs. Cette observation participante a été utilisée comme "une opportunité de construire une relation de confiance qui constituera [a constitué] la clef d'accès aux données » (THIÉTART, 2003, p. 242).

\section{Les entretiens semi-directifs}

L'enquête par entretiens représente la méthode principale de recueil de données pour cette recherche. Tous les acteurs de l'entreprise Delta - dirigeant, associés, employés - ont été interrogés ainsi que deux personnes en lien avec la gestion de l'entreprise (l'expert-comptable et l'assureur), soit treize individus. Ils ont été interviewés durant le mois de février 2006, selon un mode semi-directif (BERTHIER, 1998) sur les thèmes suivants:

- les dynamiques organisationnelle, de production et environnementale (de réseau) de Delta;

- les logiques d'action des acteurs de l'entreprise Delta.

Lors de ces entretiens, d'une durée moyenne d'1 heure à une 1 heure 30(5), le chercheur a veillé à établir un rapport d'empathie avec les acteurs interrogés (KAUFMANN, 1996) qui "permet d'"entrer" dans les logiques particulières de ceux-ci et de la [les] comprendre de l'intérieur " (FriedberG, 1993, p. 305). Ces entretiens ont fait l'objet d'un enregistrement numérique (avec l'accord préalable des acteurs) et ont tous été intégralement retranscrits.

(1) Qui s'observe notamment (au regard de nombreux indicateurs quantitatifs et qualitatifs) par une augmentation de sa production et de ses ressources humaines, financières et matérielles, par une diversification de ses produits, par un début de structuration de ses fonctions de gestion (PAGET, 2007).

(2) Pour commercialiser ce type de produits dans le respect de la législation en vigueur (c'est-à-dire au moment de l'enquête, la loi $\mathrm{n}^{\circ}$ 92-645 du 13 juillet 1992), l'entreprise possède une licence d'agent de voyages. À noter que la législation a depuis évolué (loi $\mathrm{n}^{\circ} 2009-888$ du 22 juillet 2009 de développement et de modernisation des services touristiques).

(3) Le terme «séminaire» est une appellation générique utilisée par les acteurs du secteur pour désigner tout type de manifestations à caractère professionnel.

(4) Afin de pouvoir proposer à la vente des activités sportives (de l'encadrement sportif), l'entreprise est déclarée établissement sportif auprès des services concernés. Ceci constitue une obligation réglementaire en application de l'article L. 322-3 du Code du sport, du décret n 93-1101 du 3 septembre 1993 et de l'arrêté du 27 juin 2005.

(5) Â l'exception du dirigeant pour lequel trois entretiens ont été nécessaires, d'une durée totale d'environ quatre heures. 


\begin{abstract}
Les documents
Parallèlement à l'enquête par entretiens et à l'observation participante, le chercheur a procédé à la collecte de tous les documents pouvant aider à la compréhension de l'action organisée (FriedberG, 1993). Ces écrits (statuts de l'entreprise, registre du personnel, documents financiers, documents relatifs à la production, communication interne...) ont permis d'éclairer et de vérifier (dans certains cas) les propos des acteurs, ainsi que d'apporter des preuves pour étayer l'analyse et valider la recherche.
\end{abstract}

L'ensemble des données récoltées a fait l'objet d'une analyse de contenu de type thématique (BARDIN, 1993).

secteur du sport et du tourisme. Pourtant ce dernier constitue un contexte particulier pour les entreprises s'y insérant. Il leur confère des caractéristiques propres liées à la nature même du service sportif et touristique (PAGET, 2007). Celles-ci s'observent en termes de saisonnalité du métier, de formation du dirigeant (issu de pratiques de loisir et d'un apprentissage personnel), de motivations de création de l'entreprise, de la recherche d'un développement personnel, des valeurs relatives au sport et au loisir, et enfin, d'un réseau de partenaires (indispensable à la réalisation de produits multi-activités ou à forfait).

Il est donc intéressant de comprendre comment ces entreprises s'adaptent à leur environnement et mettent en œuvre des stratégies particulières notamment de flexibilité. Pour cela, nous nous appuierons sur une analyse organisationnelle entendue au sens large. Pour Bernoux (2004), les différentes approches sociologiques des organisations se complètent et peuvent être mobilisées tour à tour pour rendre compte de la diversité et de la complexité des phénomènes organisationnels. Ainsi, au cours de la réflexion, il sera fait appel aux concepts les plus pertinents et les plus à même d'expliquer les processus à l'œuvre. Il s'agira bien d'appréhender les mécanismes de cette flexibilité, c'est-à-dire le processus par lequel une entreprise peut s'adapter à son secteur, anticiper des fluctuations, ou encore se créer des opportunités lui permettant de croître. Ainsi, cet article expliquera, à partir d'une monographie et d'un point de vue stratégique, comment un dirigeant peut parvenir à rendre son entreprise flexible et il s'intéressera à l'adhésion des employés à ce type de gestion ( $c f$. encadré 1$)$.

Dans un premier temps, nous dégagerons les particularités du secteur et les stratégies mises en œuvre par le dirigeant de l'entreprise étudiée pour y faire face, en termes de gestion des emplois. Nous mettrons en évidence, dans un second temps, la non-formalisation de l'entreprise comme mode de gestion à part entière et ses implications. Enfin, nous montrerons que bien que les incertitudes du secteur soient reportées sur les employés (variables d'ajustement), ces derniers adhèrent au «projet d'entreprise» du dirigeant. Les déterminants de cette coopération des acteurs seront mis au jour.

\section{Rythmes touristiques et gestion des emplois}

Delta évolue dans un environnement présentant des spécificités qui exercent sur l'entreprise de fortes contraintes. Pour y faire face, l'analyse montre que le dirigeant joue sur les frontières organisationnelles de son entreprise.

\section{Les particularités du secteur: variations cycliques et acycliques}

Le secteur dans lequel opère Delta contraint et conditionne pour une part l'activité de l'entreprise, et par là même sa croissance. Les aléas climatiques et saisonniers qui lui sont inhérents jouent en effet un rôle important dans le sens où ils font peser de nombreuses incertitudes sur l'entreprise.

Le positionnement de Delta sur le créneau du tourisme d'affaires entraîne une saisonnalité de la production. La clientèle d'entreprises visée par ce type de tourisme implique cet état de fait. Les séminaires à l'initiative de leur direction et à l'attention de leur personnel (voire parfois de leurs clients, collaborateurs ou de journalistes), se déroulent exclusivement sur le temps de travail de l'entreprise, donc pendant des jours ouvrables. Les weekends et périodes de vacances scolaires en sont ainsi exclus. De plus, la société Delta étant une agence réceptive de tourisme spécialiste de la montagne (de par son implantation en station de sports d'hiver), les demandes de sa clientèle d'entreprises pour la réalisation de séminaires se concentrent massivement sur la saison hivernale.

Ce pic hivernal d'activité est également lié à la période enneigée, mais la fréquentation touristique en station de sports d'hiver est variable d'une année à l'autre, notamment avec les problèmes de manque de neige (ODIT, 2006). De plus, Delta est soumis aux aléas climatiques du milieu montagnard puisque le temps ne permet pas toujours la réalisation de ses produits.

Ainsi l'entreprise prestataire de sport et de tourisme en montagne doit être capable de s'adapter aux variations cycliques et acycliques de son environnement, à savoir une double saisonnalité, des flux touristiques aléatoires et des conditions climatiques particulières. 


\section{Des frontières organisationnelles floues}

Pour faire face à la spécificité du secteur, le dirigeant de l'entreprise Delta met en œuvre une stratégie particulière (CROZIER, FrIEDBERG, 1977). Il joue sur tous les statuts possibles d'emploi. Il en résulte que sa société n'est pas une organisation figée: ses frontières et sa structure sont au contraire floues et poreuses.

Il s'entoure tout d'abord d'un noyau dur de salariés permanents, embauchés en contrat à durée indéterminée (CDI), qui lui permettent d'assurer la pérennité de son entreprise. Aucun de ces employés n'est cadre sportif. Tous ont été recrutés sur des compétences en lien avec le métier d'agence de voyages de Delta, c'est-à-dire d'assemblage de produits touristiques (il s'agit par exemple de chefs de projets, de responsables administratifs, de production). Parallèlement, le dirigeant a recours de manière systématique à de la main-d'œuvre périphérique. D'une part, il fait appel à des personnes qu'il embauche en contrat à durée déterminée (CDD) (2). Celles-ci sont recrutées au gré des besoins de l'entreprise, soit à la saison, soit pour une mission particulière, soit même pour le temps d'une soirée (ce peut être par exemple des assistants administratifs, des animateurs, des pisteurs secouristes). D'autre part, pour le métier de producteur d'activités de Delta, le dirigeant fait appel à des cadres sportifs extérieurs à son entreprise, travailleurs indépendants, ce statut étant caractéristique du milieu sportif et montagnard. Ces acteurs ont une compétence sportive (par exemple moniteur de plongée ou accompagnateur moyenne montagne) qui est par la suite (pour les besoins de Delta) élargie au sein de l'entreprise en une compétence technique de montage d'activités et de production non plus seulement sportive. Le dirigeant recrute ainsi dans son réseau de connaissance sportif, du personnel qu'il n'a pas besoin de salarier. Enfin, l'entreprise Delta accueille un nombre important de stagiaires (proportionnellement à son nombre d'employés permanents (3)). Ceux-ci sont recrutés tout au long de l'année (et non pas seulement en période de pic d'activité) pour des missions diverses (par exemple pour effectuer un travail de chef de projet ou des tâches de technicien).

Cette main-d'œuvre secondaire (BALLOT, 1996; LAZEAR, 1998) constitue une variable d'ajustement pour le dirigeant de Delta: il l'utilise pour

(2) Il nous faut préciser que Delta est rattaché à la convention collective nationale des agences de voyages et de tourisme du 12 mars 1993 (étendue par arrêté du 21 juillet 1993 JORF du $1^{\text {er }}$ août 1993). Le recours aux CDD (saisonniers) est possible de manière régulière dans ce cadre.

(3) En 2006 par exemple, le nombre de stagiaires intervenus a été de quatre (représentant une durée cumulée dans l'entreprise de onze mois) pour dix employés permanents; en 2003, ils étaient déjà au nombre de quatre (représentant une durée cumulée dans l'entreprise de dix-huit mois) mais pour trois employés en CDI. gérer les aléas du secteur. Cette forme de flexibilité de l'entreprise, à la fois externe et quantitative, consiste à puiser dans l'environnement pertinent les ressources humaines nécessaires et à ajuster le nombre de salariés en fonction de la demande; elle permet ainsi à Delta d'amortir les fluctuations du marché. Cependant, cette flexibilité présente quelques limites.

La première provient d'une particularité de l'entreprise Delta. Ses «employés»(4) temporaires (lorsqu'ils interviennent au moins une semaine dans l'entreprise) ne viennent pas en remplacement du personnel en CDI (qui serait en congé ou qui ne souhaiterait pas effectuer d'heures supplémentaires) comme l'ont montré JACQUOT et SETTI (2002). Ils font partie à part entière de l'entreprise. Pour preuve, Delta intègre comme responsable de service ou de site (station de sports d'hiver), certains de ces travailleurs indépendants. Le responsable du service technique par exemple (en lien avec le métier de producteur d'activités de l'entreprise) est un travailleur indépendant, qui n'est donc pas salarié de Delta. Mais comme il n'est pas non plus considéré comme un sous-traitant, il constitue bien un maillon de l'entreprise. Plusieurs indicateurs attestent de cette intégration: courriel au nom de Delta au même titre que les employés en CDI, bureau personnel au sein des locaux de l'entreprise, téléphone de fonction(5). Cela signifie que cet acteur est un responsable d'une organisation à laquelle il n'appartient pas; il n'existe aucun lien de subordination entre le dirigeant de Delta et ce travailleur indépendant (engagé à la tâche et payé sur facture).

Seconde limite, la possibilité pour l'entreprise Delta d'avoir recours à une main-d'œuvre extérieure au gré de ses besoins est à nuancer. La flexibilité dans le secteur du sport et du tourisme n'est pas aisément réalisable à tous les niveaux. En effet, si le métier de producteur d'activités (assumé au sein de Delta par des cadres sportifs) se prête bien à l'embauche de salariés temporaires de par la nature des compétences requises (à la fois sportive et de manutention) et la disponibilité d'un nombre important de travailleurs indépendants en station de sports d'hiver, ce n'est pas le cas du métier d'assembleur

(4) Lorsque le terme «employés» est mis entre guillemets dans le texte, celui-ci devra être compris comme l'ensemble des acteurs de l'entreprise Delta (à l'exception du dirigeant). À ce titre, les travailleurs indépendants y sont inclus.

(5) Il faut indiquer qu'il ne s'agit pas là d'un montage financier mais que cette situation résulte d'une évolution de l'entreprise. Cette intégration de l'acteur a bien commencé de manière ponctuelle ou saisonnière: le travailleur indépendant était responsable technique au sein de Delta lors de la saison hivernale; le reste de l'année, il pouvait être (pour son compte) pilote de montgolfière, moniteur de plongée ou autre. Ainsi les deux parties (le dirigeant de Delta et le travailleur indépendant) gardaient bien chacune leur indépendance vis-à-vis de l'autre. La relation s'est progressivement transformée et s'est étendue de plus en plus au reste de l'année, du fait de la croissance de Delta. 
touristique. L'organisation de séminaires implique un travail de conception de produits sur mesure pouvant difficilement être accompli par un individu embauché ponctuellement. Deux raisons l'expliquent: d'une part l'entreprise, comme la plupart des petites entreprises du secteur, est peu formalisée, ce qui implique qu'il n'existe pas de standardisation des tâches et des procédures; et d'autre part, le travail d'un «chef de projet» nécessite une connaissance fine des produits et des activités proposés par Delta. La fonction liée à l'activité d'agent de voyages requiert donc un apprentissage au sein de l'entreprise.

\section{L'informel, un mode de gestion à part entière}

Une régulation de contrôle (REYNAUd, 1999) particulière est à l'œuvre au sein de Delta: il s'agit d'une non-formalisation.

\section{Une régulation de contrôle inhabituelle}

Alors que l'entreprise a huit ans (6) et contrairement à ce que prescrivent les auteurs de la théorie de la contingence (MinTZBERG, 1982), l'informel prédomine. Le dirigeant communique très peu sur ses politiques de gestion, privilégie des process basés sur les routines organisationnelles et s'appuie sur des «contrats moraux» avec ses employés.

Toutes les fonctions de gestion de l'entreprise sont concernées et présentent un faible degré de structuration. Les politiques de gestion des emplois, de rémunération, de formation sont soit inexistantes (il n'y a par exemple aucune analyse prévisionnelle des recrutements, aucun plan de formation des salariés), soit informelles (par exemple lors d'une embauche, aucune rémunération n'est fixée sur la base du SMIC; l'augmentation des salaires, l'obtention de primes ou d'avantages en nature se font à l'appréciation du dirigeant en fonction de la croissance de l'entreprise). Par ailleurs, Delta ne réalise aucune étude de marché, les différents acteurs de l'entreprise menant leur propre analyse au contact de la clientèle et de la concurrence. Des politiques de produit, de prix, de distribution et de promotion existent mais, dans leur grande majorité, ne sont pas formalisées. Lors de la production de devis par exemple, les chefs de projet savent qu'ils doivent appliquer une marge au coût de chaque prestation ainsi qu'une marge finale mais il n'existe aucune grille tarifaire des produits, ceux-ci étant réalisés sur mesure et leurs modalités changeant donc à chaque fois. Le calcul tarifaire des prestations résulte alors d'un bricolage des acteurs comme en témoignent ces extraits d'entretiens: "Quand on voit les devis

(6) À la date des entretiens. de [employé F] qui dit ben ça, ça coûterait combien, ça, ça coûterait combien? C'est marqué nulle part, c'est [employé A] qui va dire "ben ça, ça coûte tant, ça, ce serait bien que tu fasses ça et puis tu penses à mettre une marge en plus" " [employé D]; "tu fais des prix à la louche » [employé F]. Enfin, s'il existe une formalisation de l'organisation du travail chez Delta (structuration par services: service production pour la "partie agence de voyages», service technique pour la «partie production d'activités», service commercial et service administratif; avec un responsable par service), la plupart des process de production sont informels au sein de l'entreprise. Il existe en effet un flou organisationnel par rapport aux procédures de traitement des dossiers, accentué en période de forte activité. Les méthodes de travail sont également peu systématisées. Il n'y a pas, par exemple, de modèles informatiques de document type tel que devis, confirmation de prestataires, facture; les employés sont donc obligés de refaire à chaque fois les formules de calcul, les descriptifs de prestations. Le dirigeant parle de «procédures [...] qui se transmettent», d' «automatismes» et d'une «forme d'éducation». Il explique qu' «il n'y a pas de règles, il n'y a pas d'écrits, c'est plus une manière de fonctionner ensemble». L'organisation du travail au sein de Delta n'est donc quasiment pas structurée de manière formelle mais résulte plutôt de routines organisationnelles provenant du noyau dur d'employés qui «ont engrangé des informations» [employé D].

Grâce à ce mode de fonctionnement, Delta possède une forme de flexibilité organisationnelle qui lui permet de s'adapter aux variations du secteur sportif et touristique, aux situations nouvelles et imprévues pouvant survenir. En effet, par la (quasi) non-formalisation de Delta, le dirigeant n'impose pas aux acteurs de son entreprise un mode de résolution des problèmes. Il leur laisse une marge de liberté dans leurs actions de travail. L'informel apporte ainsi de la souplesse dans la gestion en offrant aux acteurs des possibilités d'ajustement continu aux changements de l'environnement. Cela se traduit par des volumes horaires de travail très variables selon les périodes de l'année et les fluctuations du marché.

\section{La non-formalisation: une forme de pouvoir}

Cependant, nous pouvons nous demander si cette manière de gérer l'entreprise ne constitue pas également une source de pouvoir pour le dirigeant de Delta, liée à l'incertitude que la non-formalisation crée pour les «employés».

Pour bien comprendre ce phénomène, revenons tout d'abord sur la formalisation et ses implications. La règle formelle est un instrument de gouvernance visant «à rationaliser les problèmes, à faire disparaître des incertitudes et des aléas de comportement et à stabiliser, à régulariser et par là à rendre plus 
prévisible le fonctionnement d'une organisation» (FrIEDBERG, 1993, p. 156). Au sein d'une entreprise, un dirigeant la met en œuvre pour encadrer les actions de ses employés, réguler leurs conduites et leurs pratiques, délimiter leurs capacités d'action. Cependant, cette formalisation ne représente jamais qu'une photographie de l'organisation en question et non son fonctionnement réel. En effet, la régulation qu'elle opère est sans cesse dépassée et reprise par les acteurs du fait de ses effets pervers: les «employés» se saisissent des zones d'incertitude créées par les règles pour satisfaire des intérêts particuliers qui peuvent être différents de ceux de l'organisation. La régulation de contrôle habituelle (formelle) permet donc de «structurer des espaces de négociation et de jeu entre acteurs » (FriedBERG, 1993, p. 160). Elle est ainsi à la fois contrainte et partie prenante des interactions entre salariés. Mais, ces règles, qui permettent de figer le comportement des acteurs, font perdre dans le même temps du pouvoir à celui qui les crée puisqu'il devient plus prévisible. En effet, les règlements, les procédures, les buts formels... rendent le comportement du dirigeant parfaitement prévisible pour ses employés et limitent son arbitraire. La formalisation correspond donc à une situation où un acteur-dirigeant accepte de perdre du pouvoir en devenant moins incertain, pour pouvoir fixer le comportement de ses protagonistes-salariés.

Dans le cas de Delta, c'est le contraire qui s'observe. Le dirigeant conserve, grâce à l'informel, une forme d'imprévisibilité et celle-ci lui procure une source de pouvoir. La non-formalisation lui apporte une plus grande marge de manœuvre au sein de son entreprise parce qu'il est ainsi moins prévisible. Cette imprévisibilité lui permet de créer et de contrôler de l'incertitude au sein de Delta, et «ce qui est incertitude du point de vue des problèmes est pouvoir du point de vue des acteurs » (CROzIER, FRIEDBERG, 1977, p. 24). Le pouvoir est la capacité d'un acteur à faire changer le comportement d'un autre sous son influence; il s'exerce dans les relations d'échange qui, bien qu'elles soient réciproques, sont déséquilibrées. Ainsi, la non-formalisation du contexte d'action, qui signifie pour les «employés» une incertitude quant au comportement du dirigeant, confère à ce dernier une capacité de négociation avec ceux-ci. Elle lui permet de moduler ses politiques de gestion au gré des situations. En effet, en fonction des fluctuations du marché ou d'intérêts plus personnels, le dirigeant procède par exemple à des ajustements par les rémunérations: à l'embauche dans la fixation du montant du salaire, dans le versement ou non de primes, dans l'attribution ou le retrait d'avantages en nature... Il fait également varier sa politique de gestion des ressources humaines tant du point de vue des recrutements (et des contrats de travail associés) que des licenciements (les uns et les autres étant conditionnés par sa rationalité affective). Finalement, la non-formalisation de la gestion lui permet de diriger son entreprise avec, en quelque sorte, un «masque»: on ne peut lui faire de reproches sur des éléments qui ne sont pas connus, qui n'ont pas été annoncés et le dirigeant peut donc modifier les «règles du jeu» à sa guise. Ainsi, comme le soulignent les propos d'un acteur, «même s'il a tort, il a raison».

L'informel constitue donc une ressource fondamentale pour l'entrepreneur par la marge de manœuvre et les possibilités de jeu qu'il lui assure. Il lui offre un large éventail de conduites potentielles pour obtenir la coopération de ses «employés » pour la réalisation de ses objectifs et de son projet de croissance.

\section{Autonomie et implication des employés}

Le mode de gestion mis en place par le dirigeant de Delta est centré sur une flexibilité forte de l'entreprise. Le dirigeant joue sur les frontières organisationnelles de l'entreprise et privilégie une régulation de contrôle basée sur l'informel, pour faire face aux fluctuations de l'environnement sportif et touristique. Mais dans ce système, les incertitudes du secteur sont reportées sur les «employés» qui représentent une variable d'ajustement pour le dirigeant. Comment cette flexibilité est-elle ressentie par les acteurs de l'entreprise et comment y réagissent-ils? L'analyse approfondie de Delta et des logiques d'action des acteurs met en évidence une adhésion de ceux-ci au management et au projet du dirigeant, et même une perception positive de leur part. L'autonomie et l'implication des «employés » apparaissent comme les déterminants de leur coopération, indispensable à la pérennité de l'entreprise.

\section{Une distribution particulière des rôles au sein de l'entreprise}

L'entreprise Delta est très peu formalisée mais ce n'est pas par défaut. D'une part, comme nous l'avons montré supra, cette non-formalisation de l'organisation procure au dirigeant une certaine marge de manœuvre dans ses actions de gestion. D'autre part, elle lui permet dans le même temps de fournir aux acteurs de son entreprise une marge de liberté afin de poursuivre leurs objectifs et de produire leur propre régulation.

Ainsi, le dirigeant donne à ses «employés» une très large autonomie dans leur travail. L'entreprise n'a pratiquement que des «chefs» responsables de service, de site ou de projet ( $c f$. tableau 1$)$; le personnel d'exécution est donc quasi inexistant 
Tableau 1 : Organigramme de la société Delta

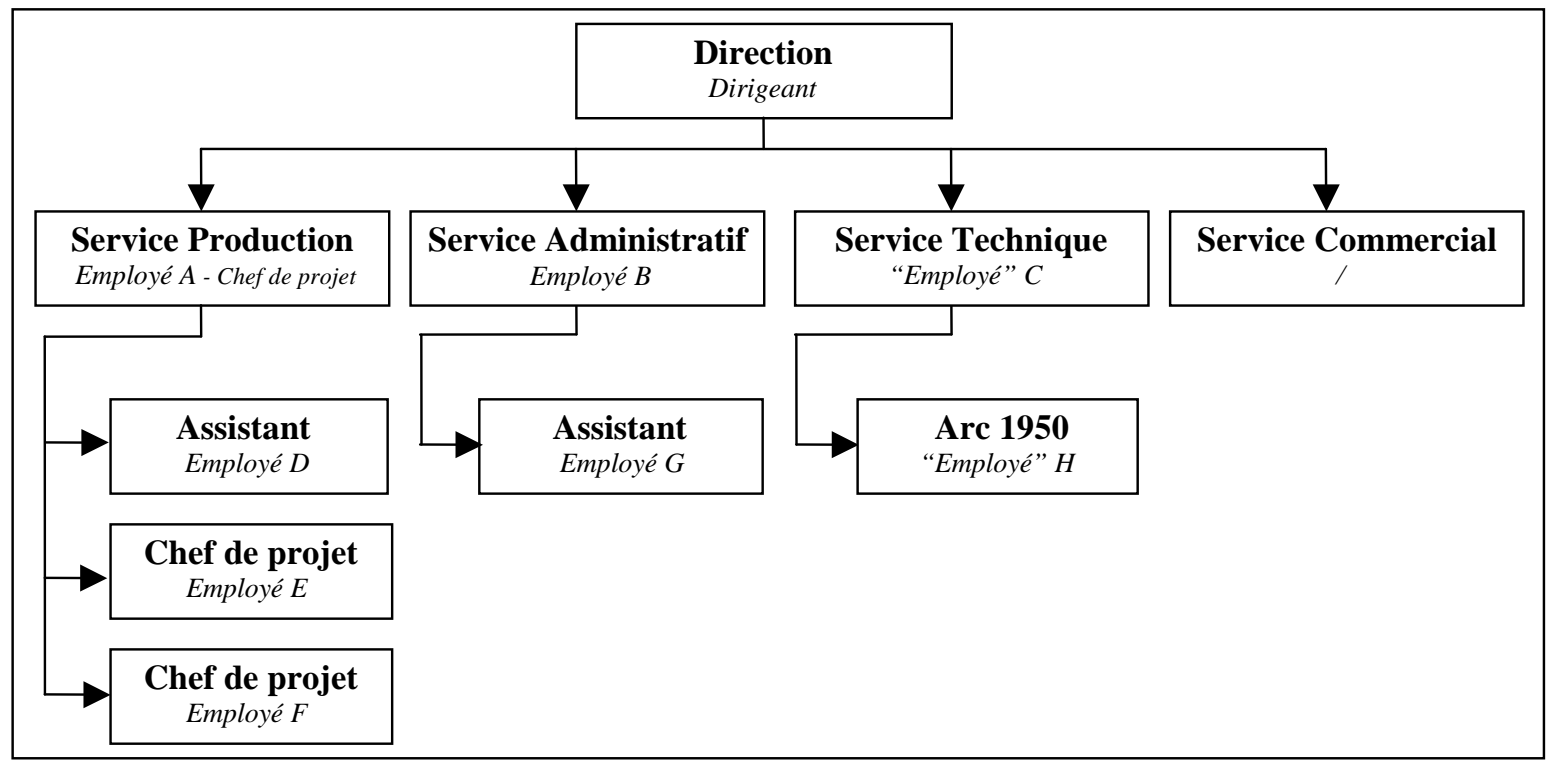

dans l'entreprise (un seul assistant de production en contrat à durée indéterminée - employé D - et un assistant administratif mais en contrat à durée déterminée seulement - employé G).

Le dirigeant a délégué la fonction production de son entreprise et a, par cette opération, responsabilisé les acteurs de son entreprise auxquels il accorde sa confiance. Chaque chef de projet est en charge de son dossier, depuis le premier devis jusqu'à la facturation après la prestation. Le dirigeant exerce un faible contrôle; le debriefing des séminaires se fait par exemple de manière informelle autour d'un café. Or, bien que cette autonomie puisse constituer une source de pouvoir pour les employés, celle-ci n'est pas utilisée comme telle. Au contraire, elle est transformée en pertinence (FRIEDBERG, 1993) par le personnel de Delta, au service de l'entreprise, dans le sens où ces acteurs ne font pas défaut et sont très présents les uns pour les autres. Le taux d'absentéisme au sein de l'entreprise reflète particulièrement ce point : il est proche de zéro; tout comme les justifications des employés: "Quand tu es malade, tu viens quand même au travail, mais tu le fais parce que tu as envie, tu n'as pas envie de laisser les autres dans la m...» [employé D]. De même, les relations interpersonnelles permanentes qu'ils entretiennent (favorisés par le fait qu'ils travaillent dans une même pièce), leur permettent de résoudre les problèmes au fur et à mesure qu'ils apparaissent, grâce à l'aide des autres, et donc de bricoler les procédures (formelles ou informelles) lorsque celles-ci présentent des failles. Ainsi, les acteurs de Delta sont tous des «chefs » mais interdépendants; et si l'entreprise peut fonctionner selon ces modalités, c'est grâce à leur régulation autonome (REYNAUD, 1999) et à leur fiabilité dans l'organisation. En effet, la confiance est une composante essentielle de cette forme de gestion par projet (BoltANSKI, CHIAPELlo, 1999) mise en place par le dirigeant et donc de la réussite de l'entreprise.

\section{Un brouillage \\ entre temps de travail et temps libre}

Les acteurs de l'entreprise Delta coopèrent à ce type particulier de gestion, axé sur la flexibilité et l'adaptabilité de l'entreprise au secteur. Les variations du marché sont reportées sur les «employés » et pourtant, ces derniers acceptent les rôles qui leur sont assignés, effectuent de très nombreuses heures de travail (au-delà de ce qui est normalement prévu dans leur contrat de travail), bricolent les règles lorsque cela s'avère nécessaire pour garantir la qualité du produit, et cela au profit de l'entreprise, afin que cette dernière puisse continuer à fonctionner et à se développer. Or, une caractéristique singulière de Delta est que cet investissement requis par le dirigeant envers ses «salariés», qui dépasse très largement ce qui est communément exigé par un employeur, n'est pas vécu par ces acteurs comme imposé; cette mobilisation est au contraire perçue de manière positive par les «employés». Les modalités de la gestion par projet mise en place au sein de Delta et l'intégration de la société du loisir par le dirigeant permettent d'en comprendre les ressorts. Ceux-ci apparaissent liés à un brouillage des temps sociaux, notamment entre temps de travail et temps libre.

\section{Les modalités de la gestion par projet}

L'autonomie et l'implication des acteurs sont deux éléments fondamentaux de l'organisation du travail dans l'entreprise, vecteurs de la réussite de Delta. Les employés de Delta acceptent de travailler sur de longs horaires, au point qu'il leur est souvent difficile de solder leurs jours de congés [employé B]. 
Cet engagement fort des acteurs provient du mode de gestion par projet qui implique une conception forfaitaire du travail, orientée vers la réalisation d'objectifs (BoufFarTigue, Bocchino, 1998) et donc déconnectée du temps de travail (BEAujolin-BELLET, 2004). La place est à l'autorégulation des ressources humaines et au contrôle autocentré (BOLTANSKI, Chiapello, 1999). Il en découle que ce qui compte dans ce type de management, ce sont moins les qualifications «objectivées» des acteurs de l'entreprise que leurs compétences. Le dirigeant s'appuie sur les «propriétés de la force de travail» c'est-àdire sur les qualités intrinsèques des «employés», sur leur capacité à s'adapter, à s'impliquer, sur leurs aptitudes relationnelles. Il accorde du crédit au «savoir-être» des individus, ce qui est à mettre en lien avec le fait que les savoirs et savoir-faire ne sont que peu formalisés dans l'entreprise Delta.

Ce que ce mode de gestion induit, c'est une part de liberté accordée à l'acteur dans son travail. Responsabiliser les «employés» signifie leur fournir une marge de manœuvre qui leur permette de laisser s'exprimer leur créativité et leur esprit d'initiative. Le dirigeant joue ainsi sur le développement personnel des acteurs de son entreprise en leur offrant une forme d'épanouissement au travail. Dans ce système, la référence au client sert de moyen d'évaluation des «employés». La satisfaction du client détermine la ligne de conduite au sein de l'entreprise et constitue le «projet collectif» garantissant le succès de l'entreprise. Par cette démarche, une part du contrôle des «employés » est donc transférée au client.

Ce fonctionnement managérial, en favorisant l'autonomie et par là, l' autoréalisation de chacun des acteurs, a donc entraîné un effacement de la distinction entre vie privée et vie professionnelle chez les «employés», et donc entre travail et non travail, entre intéressement et désintéressement (BOLTANSKI, Chiapello, 1999). Il a permis de faire converger les acteurs de Delta dans un secteur mouvant, d'encourager leur créativité, leur réactivité, leur flexibilité, et de brouiller finalement les repères entre sphères privée et professionnelle.

\section{L’intégration de la société du loisir}

Mais le brouillage des temps sociaux ne provient pas seulement de la forme de gestion par projet mise en place au sein de Delta. Le dirigeant va encore plus loin pour intéresser et enrôler les acteurs (CALLON, 1986; LATOUR, 2006) dans le projet de croissance qu'il a pour son entreprise: il intègre la société du loisir dans son entreprise.

Le système de contribution - rétribution qu'il a instauré avec ses «salariés» n'est pas uniquement fondé sur du matériel, sur de l'utilitaire, mais se compose également d'une rémunération symbolique. En effet, le dirigeant assure un bon salaire à ses employés et y ajoute même des avantages en nature (qui peuvent être une voiture de fonction, un téléphone portable, des indemnités de logement...); mais il leur offre en plus du loisir (Pronovost, 1997). Par exemple, cela se traduit par un forfait de ski à la saison offert à tous les «employés » (7). En contrepartie de ces différents avantages, les acteurs s'investissent complètement dans l'entreprise et dans le projet du dirigeant en ne comptant pas leurs heures de travail et se montrant fiables dans l'organisation. Le dirigeant arrive là encore à estomper la frontière entre les espaces temporels dévolus au travail et ceux réservés au temps de loisir de ses «employés » (Viard, Potier, Urbain, 2002). À l'unanimité, ces derniers déclarent aimer leur travail, sa diversité et les horaires non conventionnels qui lui sont inhérents: «J'aime mon métier», «on aime tous notre boulot», «l'esprit [d'équipe n'est] pas seulement au travail», «on ne fait jamais la même chose»...; leurs discours montrent une absence de rupture nette entre leur travail et leur loisir. De plus, pratiquement tous les acteurs de l'entreprise expriment leur croyance en Delta et en son succès ( «j'y crois », «une structure en laquelle je crois»...), et même parfois leur fierté de travailler pour cette entreprise de par l'image de réussite qu'elle véhicule. Les «employés» se sentent engagés dans un projet (de croissance), dans une «aventure commune». Le dirigeant fait participer les acteurs de son entreprise à un «rêve» (Boltanski, Chiapello, 1999).

Mais cet «enrôlement» des acteurs (AKRICH, Callon, Latour, 2002) est rendu possible par une personnalisation des recrutements (COMBES, 2002). Les acteurs de Delta ne sont en effet pas là par hasard: le dirigeant "pré-teste» ses employés en les embauchant d'abord en stage ou en CDD dans son entreprise. Très peu d'employés permanents ont été directement embauchés en CDI et sur les quatre seuls recrutements effectués sur ce mode, deux se sont avérés un échec pour le dirigeant puisque l'issue a été la démission de l'employé, celui-ci n'adhérant pas à la «culture» de Delta. Cette méthode de recrutement permet au dirigeant de s'assurer, sur une période limitée, de la bonne adéquation des employés avec son entreprise et son mode de gestion. La sélection des acteurs s'appuie sur des justifications en valeurs (BOLTANSKI, THÉVENOT, 1991) qui privilégient ( $c f$. supra) les «compétences personnelles » et l' «état d'esprit» [dirigeant] de la personne. Cette personnalisation permet en effet à l'acteur-dirigeant de "garantir la source d'une expérience commune, de référents professionnels, culturels et psychologiques semblables» et provient probablement de «l'incapacité à nommer les compétences requises» (ComBEs, 2002, p. 17) pour les différents postes de travail.

(7) Rappelons que l'entreprise Delta est implantée en station de sports d'hiver. 


\section{* \\ $* *$}

Cette étude a permis, outre un apport de connaissances sur le secteur sportif et touristique, de comprendre comment la mise en œuvre d'un mode de gestion centré sur la flexibilité (assurant la croissance d'une entreprise) peut être acceptée par des employés et même les satisfaire. En observant de plus près le jeu organisationnel au sein de l'entreprise étudiée, il peut être mis en évidence des avantages et des inconvénients de part et d'autre. D'un côté, le dirigeant de Delta se prive des moyens d'une régulation formelle, c'est-à-dire d'un instrument de gouvernement qui lui permettrait de «fixer» le comportement des «employés» et de les faire rentrer dans le monde de l'industrie (BolTANSKI, THÉVEnOT, 1991) régi par l'efficacité. Mais, il obtient en contrepartie de la flexibilité organisationnelle ainsi que dans ses politiques de gestion des ressources humaines. Il y gagne la paix sociale, une forte croissance de son chiffre d'affaires et le maintien d'une masse salariale faible. D'un autre côté, les «employés» obtiennent une autonomie dans leur travail, du rêve (en lien avec la culture de l'entreprise) et une part de loisir (épanouissement personnel), en plus d'une rémunération matérielle de base non négligeable. Cependant, ils y perdent en retour une partie de leur temps privé (en ne comptant pas leurs heures) et de leur temps de travail (lié à un manque de rationalisation des méthodes et des tâches), et évoluent également de manière aléatoire dans l'entreprise selon une reconnaissance (une évaluation) fondée sur l'arbitraire. Ainsi, la flexibilité induit des inconvénients de part et d'autre, mais doit être mise au regard (dans le secteur du sport et du tourisme) de tout ce qu'elle apporte à la fois aux employés (bénéfices économiques et symboliques) et au dirigeant (marge d'action et succès de l'entreprise).
Néanmoins, la question de la viabilité d'un tel système peut être posée. En effet, celui-ci comporte des limites - manque de rigueur apparent, flou organisationnel induisant des problèmes de coordination, horaires et charges de travail très importants - et semble difficilement tenable sur le long terme pour les employés, comme l'illustre cet extrait d'entretien: "Quand la question m'est posée "est-ce que... ce que tu fais te plait?", je dis "oui mais ça m'a usé, j'ai pris 10 ans". [...] C'est un peu délicat quand même, on va peut-être pas se multiplier par 10 tous les ans juste parce qu'on aime bien ce qu'on fait». L'enjeu pour le dirigeant semble de maintenir la flexibilité liée à la non-formalisation de l'organisation mais tout en apportant une aide aux employés. Il ne s'agit donc pas de bureaucratiser Delta, c'est-à-dire de formaliser l'entreprise et ainsi de «figer» les acteurs en leur retirant leur autonomie, parce que cela aurait pour conséquence une démobilisation et un désengagement. L'objectif serait plutôt de s'orienter vers une autonomie soutenue (COMBEs, 2002). L'organisation doit fournir un appui au personnel par un préformatage des tâches routinières. De plus, l' «employé » doit également être mobilisé à la hauteur de son niveau de compétences et l'entreprise doit pour cela lui fournir des forces vives (de l'exécutif) pour effectuer certaines tâches qu'un salarié moins qualifié pourrait assurer. Cela aura pour conséquence de diminuer la charge de travail de l'acteur. L'organigramme de la société montre que la structure est prête à les accueillir, mais qu'il n'y a pour l'instant que des « chefs $» . .$.

\section{Bibliographie}

Akrich M., Callon M., Latour B. (2002), "The Key to Success in Innovation", International Journal of Innovation Management, 6, 187-225.

Ballot G. (1996), Les marchés internes du travail: de la macroéconomie à la microéconomie, Paris, PUF/ Economica.

Barbier J.-C., NAdel H. (2003), «La flexibilité du travail», in J. Allouche et al. (eds.), Encyclopédie des ressources humaines, Paris, Vuibert.

BARDIN L. (1993), L'analyse de contenu, Paris, PUF.

BeAujolin-Bellet R. (2004), Flexibilités et performances. Stratégies d'entreprises, régulations, transformations $d u$ travail, Paris, La Découverte.
Beaujolin-Bellet R. (2003), «Les temps modernes de la préférence pour la flexibilité externe de l'emploi», in Allouche J. et al. (eds.), Encyclopédie des ressources humaines, Paris, Vuibert.

Bernoux P. (2004), Sociologie du changement dans les entreprises et les organisations, Paris, Le Seuil.

BERTHIER N. (1998), Les techniques d'enquête: méthode et exercices corrigés, Paris, Armand Colin.

Boltanski L., Chiapello E. (1999), Le nouvel esprit du capitalisme, Paris, Gallimard.

Boltanski L., Thévenot L. (1991), De la justification. Les économies de la grandeur, Paris, Métaillé. 
Bouffartigue P., Bocchino M. (1998), «Travailler sans compter son temps? Les cadres et le temps de travail», Travail et Emploi, 74, pp. 37-50.

BunEL M. (2006), «L'utilisation des modes de flexibilité par les établissements français », Travail et Emploi, 106, pp. 7-23.

Brunhes B., Kaisergruber D. (1997), Négocier la flexibilité. Pratiques en Europe, Paris, Éditions d'Organisation.

Callon M. (1986), «Éléments pour une sociologie de la traduction. La domestication des coquilles Saint-Jacques et des marins pêcheurs dans la baie de Saint-Brieuc», L'Année sociologique, 36, pp. 169-208.

Combes M.-C. (2002), «La compétence relationnelle: une question d'organisation», Travail et Emploi, 92, pp. 5-18.

CARnet de route Pour la montagne (2001), De l'écoute des clients à l'action marketing, Paris, AFIT.

Chifflet P. (1999), Sociologie politique et économie du sport, Grenoble, université Joseph-Fourier.

Clément J.-P., Defrance J., Pociello C. (1994), Sports et pouvoirs au XX' siècle, Grenoble, PUG.

Crozier M. (1964), Le phénomène bureaucratique, Paris, Le Seuil.

Crozier M., Friedberg E. (1977), L'acteur et le système. Les contraintes de l'action collectives, Paris, Le Seuil.

Everaere C. (1999), «Emploi, travail et efficacité de l'entreprise: les effets pervers de la flexibilité quantitative», Revue française de gestion, 124, pp. 5-21.

FRIEDBERG E. (1993), Le pouvoir et la règle: Dynamiques de l'action organisée, Paris, Le Seuil.

Glaser B.G., Strauss A.L. (1968), The discovery of grounded theory, Weidenfels and Nicolson, Londres.

Jacquot L., Setti N. (2002), «Réduction du temps de travail et pratiques de gestion des ressources humaines », Travail et Emploi, 92, pp. 115-131.

Kaufmann J.-C. (1996), L'entretien compréhensif, Paris, Nathan.

Latour B. (2006), Changer de société: Refaire de la sociologie, Paris, La Découverte.

Lazear E. (1998), Personnel economics for managers, New York, Edition Wiley.

Leblanc M. (1992), Le club de l'an 2000, Paris, INSEP Publications.

Loret A. (1995), Génération glisse, Paris, Autrement.

Maurice A. (1987), Le surfeur et le militant. Valeurs et sensibilités politiques des jeunes, en France et en Allemagne, des années 60 aux années 90, Paris, Autrement.

Miles M.-B., Huberman A.-M. (2003), Analyse des données qualitatives ( $2^{\mathrm{e}} \mathrm{ed}$.), Paris, De Boeck Université.

Mintzberg H. (1982), Structure et dynamique des organisations, Paris, Éditions d'Organisation.

ODIT (2006), Bilan des investissements réalisés en 2006 dans les domaines skiables alpins et sites nordiques français, Paris, ODIT France.

PAget E. (2007), L'essor d'une petite entreprise prestataire de sport et de tourisme: complexité du secteur, logiques d'action et déterminants de sa croissance, thèse de doctorat, Grenoble, université Joseph-Fourier.

Paget E., Mounet J.-P., Guilhon A. (2007), «Les petites entreprises prestataires de sport et de tourisme et leurs dirigeants: spécificités et stratégies », Revue européenne de management du sport, 17, pp. 1-13.

Pociello C. (1999), Sport et sciences sociales: histoire, sociologie et prospective, Paris, Vigot.

Pociello C. (1995), Les cultures sportives, Paris, PUF.

Pronovost G. (1996), Sociologie du temps, Bruxelles, De Boeck.

Pronovost G. (1997), Loisir et société. Traité de sociologie empirique (2 $2^{\mathrm{e}}$ ed.), Québec, Presses de l'université du Québec.

Reynaud J.-D. (1999), Le conflit, la négociation et la règle ( $2^{\mathrm{e}} \mathrm{ed}$.), Toulouse, Octarès.

ThiÉtART R.-A., (ed.), (2003), Méthodes de recherche en management ( $2^{\mathrm{e}}$ ed.), Paris, Dunod.

TARondeau J.-C. (1999), «Approches et formes de la flexibilité», Revue française de gestion, 123, pp. 66-71.

Ulrich V. (2003), «Durée annuelle du travail et outils de flexibilité du temps de travail en $2001 »$, Premières Informations et Premières Synthèses, 33 (1), pp. 1-8.

Urbain J.-D. (2002), L'idiot du voyage. Histoires de touristes, collection «Petite Bibliothèque Payot», Paris, Payot.

VIARD J. (2006), Court traité sur les vacances, les voyages et l'hospitalité des lieux, collection «Aube Poche», La Tour d'Aigues, Éditions de l'Aube.

Viard J., Potier F., Urbain J.-D. (2002), La France des temps libres et des vacances, La Tour d'Aigues, Éditions de l'Aube.

Weber M. (1992), Essais sur la théorie de la science, Paris, Agora/Presses Pocket.

YIN R. (2003), Case Study Research. Design and Methods ( $3^{\text {rd }}$ ed.), Thousand Oaks, CA, Sage. 